\title{
О критерии паранормальности для $n$-значных логических матриц
}

\author{
Наталья Евгеньевна Томова \\ Институт философии РАН. \\ Российская Федерация, 109240, г. Москва, ул. Гончарная, д. 12, стр. 1. \\ E-mail: natalya-tomova@yandex.ru
}

Аннотация: В результате обобщения на $n$-значный случай алгоритма конструирования литеральных паранепротиворечивых/параполных логик посредством комбинирования изоморфов классической логики получаем классы паранепротиворечивых, параполных и паранормальных логик. Паранормальные логики - логики, которые одновременно и паранепротиворечивы, и параполны. В качестве критерия паранепротиворечивости логики взята неверифицируемость закона Дунса Скота в соответствующей логической матрице. В качестве критерия параполноты логики взята неверифицируемость закона Клавия в соответствующей логической матрице. В статье рассмотрен тип $n$-значных логических матриц, определеяющих паранормальные системы. Исследован вопрос о классе тавтологий, определяемом этим типом матриц. Доказано, по классу тавтологий исследуемые матрицы совпадают с представленными в литературе четырехзначными паранормальными матрицами логик $\mathbf{V}, \mathbf{I}^{1} \mathbf{P}^{\mathbf{1}}$.

Ключевые слова: паранепротиворечивость, параполнота, паранормальность, $n$-значные логические матрицы, изоморфы

Для цитирования: Томова H.E. О критерии паранормальности для $n$-значных логических матриц // Логические исследования / Logical Investigations. 2021. Т. 27. № 2. C. 121-132. DOI: 10.21146/2074-1472-2021-27-2-121-132

\section{1. Введение}

Данная статья продолжает цикл работ, посвященных рассмотрению литеральных паранепротиворечивых / параполных логик ${ }^{1}$. В предыдущей статье |Томова, 2020| было построено обобщение на $n$-значный случай алгоритма построения LPP-логик (литеральных паранепротиворечи-

\footnotetext{
${ }^{1}$ T.e. в этих логиках свойства паранепротиворечивости и (или) параполноты имеют место только на уровене литералов. Литералами называем множество Lit всех формул вида $\neg^{k} p$, где $\neg^{0} p=p$ и $\neg^{k+1} p=\neg\left(\neg^{k} p\right)$, для $p \in \operatorname{Var}$, Var - счетное множество пропозициональных переменных.

(C) Томова H.E.
} 
вых/параполных логик) посредством комбинирования изоморфов классической логики. Выделены типы $n$-значных паранепротиворечивых и параполных логических матриц, порождающих те же логические теории, что и паранепротиворечивая логика Сетте $\mathbf{P}^{1}$ и дуальная ей параполная логика $\mathbf{I}^{1}$.

В настоящей статье будет указан критерий для паранормальных логических матриц, а также рассмотрен вопрос о классе тавтологий, индуцируемом этим типом матриц.

В изложении материала будем придерживаться следующего порядка. Для удобства восприятия материала вначале приведем используемые определения. Далее, поскольку мы будем рассматривать паранормальные матрицы, полученные методом комбинирования изоморфов классической логики, напомним также этот алгоритм построения LPP-логик. В следующем разделе рассмотрим четырехзначные LPP-логики. Четырехзначные логические матрицы - минимально возможные характеристические матрицы для паранормальной теории. И далее перейдем к рассмотрению $n$-значных паранормальных логик. Приведем тип соответствующих логических матриц. Докажем утверждение относительно класса тавтологий, порождаемого этими матрицами.

\section{2. Определения}

Определение 1. Пусть $\operatorname{Var}=\{p, q, r \ldots\}$ - счетное множество пропозициональных переменных и $C o n=\left\{F_{1}, \ldots F_{n}\right\}$ - конечное множество пропозициональных связок, где каждой связке $F_{i}$ сопоставлено натуральное число $a\left(F_{i}\right)$, которое обозначает число ее аргументов. Хотя бы для одного $i \in\{1, \ldots n\}$ имеет место $a\left(F_{i}\right) \neq 0$. Множество For определяется индуктивно:

(1) Var $\subseteq$ For;

(2) для каждого такого $F_{i} \in C o n$, что $a\left(F_{i}\right)=k, F_{i}\left(A_{1}, \ldots, A_{k}\right) \in F o r$, если $A_{1}, \ldots, A_{k} \in$ For;

(3) ничто иное не принадлежит For.

Алгебру формул $\mathcal{L}=\left\langle F o r, F_{1}, \ldots, F_{m}\right\rangle$ будем называть пропозициональным языком.

Пусть $\mathcal{A}=\left\langle V, f_{1}, \ldots, f_{m}\right\rangle$ - алгебра того же типа, что и пропозициональный язык $\mathcal{L}$, где $V$ - множество истинностных значений и $f_{i}-$ функция на $V$ той же местности, что и $F_{i}$.

Определение 2. Упорядоченная тройка $\mathfrak{M}=\langle V, F, D\rangle$, где $F=\left\{f_{1}, \ldots, f_{m}\right\}, D \subseteq V-$ непустое собственное подмножество $V$, 
называется логической матриией для $\mathcal{L}$. Элементы $D$ будем называть выделенными значениями :

Определение 3. Оценкой $v$ формулы $A$ в матрице $\mathfrak{M}$ для языка $\mathcal{L}$ называется такое отображение $\mathcal{L}$ в $\mathcal{A}=\left\langle V, f_{1}, \ldots, f_{m}\right\rangle$, что

1) если $p-$ пропозициональная переменная, тогда $v(p) \in V$;

2) если $A_{1}, A_{2}, \ldots, A_{n}$ - формулы и $F^{n}-n$-местная связка языка $\mathcal{L}$, тогда $v\left(F^{n}\left(A_{1}, A_{2}, \ldots, A_{n}\right)\right)=f^{n}\left(v\left(A_{1}\right), v\left(A_{2}\right), \cdots, v\left(A_{n}\right)\right)$, где $f^{n}-$ функция на $V$, соответствующая $F^{n}$.

Определение 4. Некоторая формула $A$ есть тавтология в $\mathfrak{M}$ (сокращенно $\left.-\vDash_{\mathfrak{M}} A\right)$, е.т.е. для каждой оценки $v$ в $\mathfrak{M}$ верно, что $v(A) \in D$.

Определение 5. Теорией, порождаемой $\mathfrak{M}$, называем множество всех тавтологий в $\mathfrak{M}$ и обозначаем его как $E(\mathfrak{M})$.

Определение 6. Формула $B$ логически следует из множества формул $\Gamma=\left\{A_{1}, A_{2}, \ldots, A_{n}\right\}$ в $\mathfrak{M}$ (сокращенно $-\Gamma \vDash_{\mathfrak{M}} B$ ), е.т.е. не существует такой оценки $v$ в $\mathfrak{M}$, что $v\left(A_{i}\right) \in D$ для каждой $A_{i} \in \Gamma$ и $v(B) \notin D$.

Определение 7. Отношением следования, порождаемым $\mathfrak{M}$, называем множество $C n(\mathfrak{M})$ упорядоченных пар $\langle\Gamma, B\rangle$ таких, что для всякой оценки $v$ в $\mathfrak{M}$ если $v(\Gamma) \subseteq D$, то $v(B) \in D$.

K рассмотрению матричных логик могут быть применены различные подходы (см., например, Wójcicki, 1984, ch. 2] и Девяткин, 2016, с. 30$32])$. Под логикой $L$ можно понимать пару $\langle\mathcal{L}, C n(\mathfrak{M})\rangle$, с другой стороны, логику $L$ можно рассматривать как теорию, т.е. класс тавтологий $E\left(\mathfrak{M}_{L}\right)$.

Определение 8. Изоморфом классической пропозищионалъной логики называется логическая матрица, характеризующая классический класс тавтологий.

Существуют различные формальные и содержательные критерии, характеризующие паранепротиворечивость, параполноту, паранормальность.

Мы будем использовать следующие.

Определение 9. В системе паранепротиворечивой логики не верифицируется закон Дунса Скота $A \rightarrow(\neg A \rightarrow B)$ Jaskowski, 1969 (см. также [Karpenko, 1999]).

Определение 10. В системе параполной логики не верифицируется закон Клавия $(\neg A \rightarrow A) \rightarrow A$ Ciuciura, 2015.

Определение 11. Логика называется паранормальной, если она одновременно является и паранепротиворечивой, и параполной. 


\section{3. Четырехзначные паранормальные логики}

Паранепротиворечивые и параполные логики позволяют строить выводы в условиях противоречивых и неполных данных. Для работы отдельно с каждым видом неопределенности достаточно трехзначных семантик. Однако для одновременной работы с двумя видами неопределенности необходимо как минимум четыре истинностных значения. И это понятно, так как отрицание в паранормальной матрице, с одной стороны, паранепротиворечиво, т.е. в отличие от классического отрицания оно не формирует противоречие, и формулы $A$ и $\neg A$ могут быть одновременно истинными. С другой стороны, отрицание должно обладать свойством параполноты, т.е. допускать ситуацию, чтобы формулы $A$ и $\neg A$ были одновременно ложными.

В данном разделе мы приведем ранее полученные результаты относительно четырехзначных литеральных паранормальных логик, полученных методом комбинирования изоморфов классической логики. При исследовании $n$-значных систем мы будем обращаться к этим результатам.

В статье Томова, 2018 рассмотрен класс четырехзначных литеральных паранормальных логик, полученных методом комбинирования изоморфов классической логики. Эти логики определяются логическими матрицами:

$$
\begin{aligned}
& \mathfrak{M}_{15}=\left\langle\{0,1 / 3,2 / 3,1\}, \neg_{4}, \rightarrow_{3},\{1,2 / 3\}\right\rangle ; \\
& \mathfrak{M}_{16}=\left\langle\{0,1 / 3,2 / 3,1\}, \neg_{3}, \rightarrow_{4},\{1,1 / 3\}\right\rangle,
\end{aligned}
$$

где $\neg_{3}, \neg_{4}, \rightarrow_{3}$ и $\rightarrow_{4}$ имеют следующие истинностные таблицы:

\begin{tabular}{|c|c|c|}
\hline$x$ & $\neg_{3} x$ & $\neg_{4} x$ \\
\hline 1 & 0 & 0 \\
$2 / 3$ & 0 & 1 \\
$1 / 3$ & 1 & 0 \\
0 & 1 & 1 \\
\hline
\end{tabular}

\begin{tabular}{|c|cccc|}
\hline$\rightarrow_{3}$ & 1 & $2 / 3$ & $1 / 3$ & 0 \\
\hline 1 & 1 & 1 & 0 & 0 \\
$2 / 3$ & 1 & 1 & 0 & 0 \\
$1 / 3$ & 1 & 1 & 1 & 1 \\
0 & 1 & 1 & 1 & 1 \\
\hline
\end{tabular}

\begin{tabular}{|c|cccc|}
\hline$\rightarrow_{4}$ & 1 & $2 / 3$ & $1 / 3$ & 0 \\
\hline 1 & 1 & 0 & 1 & 0 \\
$2 / 3$ & 1 & 1 & 1 & 1 \\
$1 / 3$ & 1 & 0 & 1 & 0 \\
0 & 1 & 1 & 1 & 1 \\
\hline
\end{tabular}

Доказано, что приведенные матрицы функционально эквивалентны между собой и представляют класс всех внешних четырехзначных функций ${ }^{2}$.

Также доказана функциональная эквивалентность матриц $\mathfrak{M}_{15}$ и $\mathfrak{M}_{16}$ логической матрице логики V [Puga, Da Costa, 1988] и матрице логики $\mathbf{I}_{0}$ Popov, 1999, p. 89].

\footnotetext{
${ }^{2}$ Функция $f \in F$ называется внешней, если $f\left(x_{1}, \ldots, x_{n}\right)=0$ или $f\left(x_{1}, \ldots, x_{n}\right)=1$ для любого набора истинностных значений $x_{1}, \ldots, x_{n}$.
} 
Логические матрицы логик $\mathbf{V}$ и $\mathbf{I}_{0}$ совпадают.

$\mathfrak{M}_{\mathbf{V}}=\left\langle\{0,1 / 3,2 / 3,1\}, \neg_{4}, \rightarrow_{3}, \vee_{\mathbf{V}}, \wedge_{\mathbf{V}},\{1\}\right\rangle^{3}$.

Матрица $\mathfrak{M}_{I^{1} P^{1}}$, являющаяся членом последовательности паранормальных матриц, предложенной В. Фернандесом Fernández, Coniglio, 2003 , p. 22], в точности совпадает с матрицей $\mathfrak{M}_{15}$.

Все вышеперечисленные четырехзначные паранормальные матрицы совпадают по классу тавтологий, т.е. задают одну и ту же паранормальную теорию. В [Fernández, 2001, pp. 121-123] приведена аксиоматизация логики $\mathbf{I}^{\mathbf{1}} \mathbf{P}^{\mathbf{1}}$.

\section{4. $\quad N$-значные изоморфы классической логики и LPP-матрицы}

Прежде чем перейти к рассмотрению $n$-значных литеральных паранормальных логик, кратко напомним суть метода комбинирования изоморфов классической логики для конструирования литеральных паранепротиворечивых/параполных логик (см. также [Томова, 2020, с. 150-152]).

Для построения изоморфов классической логики используются функции перевода промежуточных истинностных значений. Такая функция каждому промежуточному значению сопоставляет одно из классических значений - 0 или 1.

В случае $n$-значной логики имеем $2^{(n-2)}$ функций перевода:

1. $f_{1}(x)$ переводит все промежуточные значения $\frac{n-2}{n-1}, \ldots, \frac{2}{n-1}, \frac{1}{n-1}$ в 0 ;

2. $f_{2}(x)$ переводит все промежуточные значения $\frac{n-2}{n-1}, \ldots, \frac{2}{n-1}, \frac{1}{n-1}$ в 1 ;

3. $f_{3}(x)$ переводит промежуточные значения $\frac{n-2}{n-1}, \ldots, \frac{2}{n-1}$ в 1 и $\frac{1}{n-1}$ в 0 ;

$\cdots$

$\mathbf{2}^{(\mathbf{n}-\mathbf{2})} \cdot f_{2^{(n-2)}}(x)$ переводит промежуточное значение $\frac{n-2}{n-1}$ в 0 и промежуточные значения $\frac{n-3}{n-1}, \ldots, \frac{2}{n-1}, \frac{1}{n-1}$ в 1 .

Логическая матрица $n$-значных изоморфов классической логики выглядит следующим образом ${ }^{4}$ :

${ }^{3}$ Здесь для удобства используем нашу нотацию: истинностное значение $\overline{1}$ из Puga, $\mathrm{Da}$ Costa, 1988 в нашем обозначении $-2 / 3$ и истинностное значение $\overline{0}-1 / 3$. Матричные операции $\vee_{\mathbf{V}}$ и $\wedge_{\mathbf{V}}$ могут быть введены по определению (см. [Томова, 2018, с. 82-83]).

${ }^{4} \mathrm{~B}$ рамках нашего исследования рассматриваются логические системы, сформулированные в пропозициональном языке, включающем только лишь отрицание и импликацию в качестве исходных связок. 


$$
\mathfrak{M}=\left\langle V_{n}, \neg_{i}, \rightarrow_{i}, D\right\rangle,
$$

где $V_{n}=\left\{1, \frac{n-2}{n-1}, \ldots, \frac{2}{n-1}, \frac{1}{n-1}, 0\right\}$ - множество истинностных значений, $i=1,2, \ldots, 2^{(n-2)}, D-$ множество выделенных значений.

Используя соответствующие функции перевода, получаем $2^{(n-2)}$ отрицаний и $2^{(n-2)}$ импликаций. Примеры соответствующих таблиц истинности:

\begin{tabular}{|c|c|l|c|}
\hline$x$ & $\neg_{1} x$ & $\cdots$ & $\neg_{2}{ }^{(n-2)} x$ \\
\hline 1 & 0 & $\cdots$ & 0 \\
$\frac{n-2}{n-1}$ & 1 & $\cdots$ & 1 \\
$\frac{n-3}{n-1}$ & 1 & $\cdots$ & 0 \\
$\vdots$ & $\vdots$ & $\vdots$ & $\vdots$ \\
$\frac{2}{n-1}$ & 1 & $\cdots$ & 0 \\
$\frac{1}{n-1}$ & 1 & $\cdots$ & 0 \\
0 & 1 & $\cdots$ & 1 \\
\hline
\end{tabular}

\begin{tabular}{|c|ccccccc|}
\hline$\rightarrow_{1}$ & 1 & $\frac{n-2}{n-1}$ & $\frac{n-3}{n-1}$ & $\cdots$ & $\frac{2}{n-1}$ & $\frac{1}{n-1}$ & 0 \\
\hline 1 & 1 & 0 & 0 & $\cdots$ & 0 & 0 & 0 \\
$\frac{n-2}{n-1}$ & 1 & 1 & 1 & $\ldots$ & 1 & 1 & 1 \\
$\frac{n-3}{n-1}$ & 1 & 1 & 1 & $\ldots$ & 1 & 1 & 1 \\
$\vdots$ & $\vdots$ & $\vdots$ & $\vdots$ & $\vdots$ & $\vdots$ & $\vdots$ & $\vdots$ \\
$\frac{2}{n-1}$ & 1 & 1 & 1 & $\ldots$ & 1 & 1 & 1 \\
$\frac{1}{n-1}$ & 1 & 1 & 1 & $\ldots$ & 1 & 1 & 1 \\
0 & 1 & 1 & 1 & $\cdots$ & 1 & 1 & 1 \\
\hline
\end{tabular}

Имеем следующие логические матрицы изоморфов классической логики:

$$
\begin{aligned}
& \mathfrak{M}_{1}=\left\langle\left\{1, \frac{n-2}{n-1}, \ldots, \frac{2}{n-1}, \frac{1}{n-1}, 0\right\}, \neg_{1}, \rightarrow_{1},\{1\}\right\rangle ; \\
& \mathfrak{M}_{2}=\left\langle\left\{1, \frac{n-2}{n-1}, \ldots, \frac{2}{n-1}, \frac{1}{n-1}, 0\right\}, \neg_{2}, \rightarrow_{2},\left\{1, \frac{n-2}{n-1}, \ldots, \frac{2}{n-1}, \frac{1}{n-1}\right\}\right\rangle ; \\
& \ldots \\
& \mathfrak{M}_{2^{(n-2)}}=\left\langle\left\{1, \frac{n-2}{n-1}, \ldots, \frac{2}{n-1}, \frac{1}{n-1}, 0\right\}, \neg_{2^{(n-2)}}, \rightarrow_{2^{(n-2)}},\left\{1, \frac{n-3}{n-1}, \ldots, \frac{2}{n-1}, \frac{1}{n-1}\right\}\right\rangle .
\end{aligned}
$$

Метод комбинирования изоморфов состоит в построении такой логической матрицы, в класс матричных операций которой входят операции из двух различных изоморфов. При этом класс выделенных значений $D$ берется точно такой же, как в изоморфе, из которого взята импликация. Таким образом, применив метод комбинирования изоморфов классической логики, мы получим класс $n$-значных LPP-логик.

\section{5. $N$-значные паранормальные логики}

В статье [Томова, 2020] приведены виды $n$-значных литеральных паранепротиворечивых (и не параполных) и параполных (и не паранепротиворечивых) логических матриц, полученных методом комбинирования изоморфов классической логики. Также доказано, что эти матрицы порождают ту же паранепротиворечивую теорию, что и матрица логики $\mathbf{P}^{\mathbf{1}}$ Sette, 
1973 , и ту же параполную теорию, что и матрица логики $\mathbf{I}^{\mathbf{1}}$ Sette, Carnielli, 1995 .

Относительно класса $n$-значных литеральных паранормальных логик получены следующие результаты.

Утверждение 1. Логические матрицы вида

$\mathfrak{M}^{\text {pnorm }}=\left\langle\left\{1, \frac{n-2}{n-1}, \ldots, \frac{2}{n-1}, \frac{1}{n-1}, 0\right\}, \neg_{i}, \rightarrow_{j}, D\right\rangle$,

$$
\begin{array}{ll}
\text { (1) } \neg_{i}\left(\alpha_{k}\right)=1, & \text { (2.1) } \alpha_{k} \rightarrow_{j} x=1 \rightarrow_{j} x, \\
& (2.2) x \rightarrow_{j} \alpha_{k}=x \rightarrow_{j} 1=1, \\
& \text { (3) } \neg_{i}\left(\alpha_{m}\right)=0, \quad \alpha_{m} \rightarrow_{j} x=0 \rightarrow_{j} x=1, \\
& (4.2) x \rightarrow_{j} \alpha_{m}=x \rightarrow_{j} 0,
\end{array}
$$

где $i, j \in\left\{1,2, \ldots, 2^{(n-2)}\right\}$ и $i \neq j ; \alpha_{k}, \alpha_{m} \in\left\{\frac{n-2}{n-1}, \ldots, \frac{2}{n-1}, \frac{1}{n-1}\right\}, k \neq m$, определяют класс паранормальных логик.

Замечание 1. Условия (1) и (2) достаточны для того, чтобы закон Дунса Скота в матрице $\mathfrak{M}^{\text {pnorm }}$ не являлся тавтологией, т.е. определяют свойство паранепротворечивости матрицы.

Условия (3) и (4) достаточны для того, чтобы закон Клавия в матрице $\mathfrak{M}^{\text {pnorm }}$ не являлся тавтологией, т.е. определяют свойство параполноты матрицы.

Доказательство. Согласно определению 11, в логических матрицах, задающих паранормальные системы, не верифицируются закон Дунса Скота $A \rightarrow(\neg A \rightarrow B)$ и закон Клавия $(\neg A \rightarrow A) \rightarrow A$. Покажем, что матрица $\mathfrak{M}^{\text {pnorm }}$ удовлетворяет этому определению.

I. Докажем, что в матрице $\mathfrak{M}^{\text {pnorm }}$ закон Дунса Скота $A \rightarrow(\neg A \rightarrow B)$ не является тавтологией, т.е. существует такая оценка $v$ в $\mathfrak{M}^{\text {pnorm }}$, что $v\left(A \rightarrow_{j}\left(\neg_{i} A \rightarrow_{j} B\right)\right)=0$.

Пусть $v(A)=\alpha_{k}$ и $v(B)=0$.

Тогда $v\left(\neg_{i} A\right)=1$ (условие $\left.(1)\right)$ и

$v\left(\neg_{i} A \rightarrow_{j} B\right)=v\left(\neg_{i} A\right) \rightarrow_{j} v(B)=1 \rightarrow_{j} 0=0$.

Согласно условию (2.1), $\alpha_{k} \rightarrow_{j} x=1 \rightarrow_{j} x$. Т.к. $1 \rightarrow_{j} 0=0$, значит, $v\left(A \rightarrow_{j}\left(\neg_{i} A \rightarrow_{j} B\right)\right)=0$.

II. Докажем, что в матрице $\mathfrak{M}^{\text {pnorm }}$ закон Клавия $(\neg A \rightarrow A) \rightarrow A$ не является тавтологией, т.е. существует такая оценка $v$ в $\mathfrak{M}^{\text {pnorm }}$, что $v\left(\left(\neg_{i} A \rightarrow_{j} A\right) \rightarrow_{j} A\right)=0$. 
Пусть $v(A)=\alpha_{m}^{5}$.

Тогда $v\left(\neg_{i} A\right)=0$ (условие $\left.(3)\right)$ и

$v\left(\neg_{i} A \rightarrow_{j} A\right)=v\left(\neg_{i} A\right) \rightarrow_{j} v(A)=0 \rightarrow_{j} \alpha_{m}=1$.

Согласно условию (4.2), $x \rightarrow_{j} \alpha_{m}=x \rightarrow_{j}$ 0. Т.к. $1 \rightarrow_{j} 0=0$, значит, $v\left(\left(\neg_{i} A \rightarrow_{j} A\right) \rightarrow_{j} A\right)=0$.

Таким образом, предложенный критерий паранормальности для $n$ значных логических матриц обусловлен наличием в носителе логической матрицы как минимум двуx различных промежуточных истинностных значений таких, что если одно из них в случае отрицания ведет себя как истинностное значение 0 , а в случае импликации - как 1, то отличное от него промежуточное истинностное значение, наоборот, - в случае отрицания ведет себя как истинностное значение 1 , а в случае импликации - как 0.

Отсюда имеем:

Следствие 1. N-значная логическая матрица паранепротиворечива (закон Дунса Скота не является тавтологией), если в носителе логической матрицы имеется по крайней мере одно такое промежуточное истинностное значение, что в случае отрицания оно ведет себя как истинностное значение 0, а в случае импликации - как 1.

Следствие 2. N-значная логическая матрица параполна (закон Клавия не является тавтологией), если в носителе логической матрицы имеется по крайней мере одно такое промежуточное истинностное значение, что в случае отрицания оно ведет себя как истинностное значение 1, а в случае импликации - как 0.

Напомним, что класс литеральных паранепротиворечивых/параполных матриц, полученных методом комбинирования изоморфов классической логики, является подклассом LPР-матриц, заданных алгоритмом ЛевинаМикенберг в Lewin, Mikenberg, 2006.

Обратим внимание, что в этой же статье (р. 479) указаны условия паранепротиворечивости и параполноты для LPP-матриц. Так, LPP-матрица паранепротиворечива, если для некоторого $x \in V: x \in D$ и $\neg x \in D$. Матрица параполна, если для некоторого $x \in V: x \notin D$ и $\neg x \notin D$. Соответственно, для паранормальной LPP-матрицы необходимо наличие двух условий.

Очевидно, что условия паранормальности логической матрицы, сформулированные в утверждении 1, и вышеприведенные условия ЛевинаМикенберг согласуются между собой.

\footnotetext{
${ }^{5}$ Еще раз обратим внимание на то, что промежуточное значение $\alpha_{m}$ отлично от промежуточного значения $\alpha_{k}$.
} 
Перейдем к вопросу о паранормальной теории, задаваемой классом матриц $\mathfrak{M}^{\text {pnorm }}$.

Оказалось, что все матрицы вида $\mathfrak{M}^{\text {pnorm }}$ индуцируют ту же паранормальную теорию, что и матрицы $\mathfrak{M}_{15}, \mathfrak{M}_{16}, \mathfrak{M}_{\mathbf{V}}, \mathfrak{M}_{I^{1} P^{1}}$.

Утверждение 2. Логическая матрица вида $\mathfrak{M}^{\text {pnorm }}=\left\langle\left\{1, \frac{n-2}{n-1}, \ldots, \frac{2}{n-1}, \frac{1}{n-1}, 0\right\}, \neg_{i}, \rightarrow_{j}, D\right\rangle$,
(1) $\neg_{i}\left(\alpha_{k}\right)=1$,
(2.1) $\alpha_{k} \rightarrow_{j} x=1 \rightarrow_{j} x$
(2.2) $x \rightarrow_{j} \alpha_{k}=x \rightarrow_{j} 1=1$,
(3) $\neg_{i}\left(\alpha_{m}\right)=0$,
(4.1) $\alpha_{m} \rightarrow_{j} x=0 \rightarrow_{j} x=1$,
(4.2) $x \rightarrow_{j} \alpha_{m}=x \rightarrow_{j} 0$,

где $i, j \in\left\{1,2, \ldots, 2^{(n-2)}\right\}$ и $i \neq j ; \alpha_{k}, \alpha_{m} \in\left\{\frac{n-2}{n-1}, \ldots, \frac{2}{n-1}, \frac{1}{n-1}\right\}, k \neq m$, индуцирует ту же паранормальную теорию, что и матрица $\mathfrak{M}_{15}$.

Доказательство. Доказательство утверждения следует из фактов:

(1) если матрица $\mathfrak{M}$ является гомоморфным прообразом матрицы $\mathfrak{N}$, тогда $E(\mathfrak{M})=E(\mathfrak{N})[$ Bolc, 1992, p. 21];

(2) матрица $\mathfrak{M}^{\text {pnorm }}$ есть гомоморфный прообраз матрицы $\mathfrak{M}_{15}$ относительно отображения $h$ :

$h(x)= \begin{cases}2 / 3, & \text { если } x \in\left\{\frac{n-2}{n-1}, \ldots, \frac{2}{n-1}, \frac{1}{n-1}\right\} \text { и } \neg_{i}(x)=0, x \rightarrow_{j} y=0 \rightarrow_{i} y, \\ 1 / 3, & \text { если } x \in\left\{\frac{n-2}{n-1}, \ldots, \frac{2}{n-1}, \frac{1}{n-1}\right\} \text { и } \neg_{i}(x)=1, x \rightarrow_{j} y=1 \rightarrow_{j} y, \\ x, & \text { если } x \in\{1,0\},\end{cases}$ где $y \in\left\{1, \frac{n-2}{n-1}, \ldots, \frac{2}{n-1}, \frac{1}{n-1}, 0\right\}, k=2,3, \ldots, 2^{(n-2)}$ и $k \neq 1$.

Таким образом, мы определили класс $n$-значных литеральных паранормальных логических матриц, полученных методом комбинирования изоморфов классической логики. В ходе исследования также удалось сформулировать требования для любой $n$-значной матрицы, в которой не верифицируется закон Дунса Скота и закон Клавия. Относительно свойств теории, определяемой вышеприведенным типом матриц, показано, что этот класс матриц индуцирует ту же паранормальную теорию, что и ранее исследованные четырехзначные матрицы $\mathfrak{M}_{15}, \mathfrak{M}_{16}, \mathfrak{M}_{\mathbf{V}}, \mathfrak{M}_{I^{1} P^{1}}$ (см. |Томова, 2018|), т.е. выделен целый класс $n$-значных матриц, характеристических для этой паранормальной теории.

В результате исследован класс $n$-значных литеральных паранепротиворечивых/параполных логик, полученных методом комбинирования изоморфов классической логики. Выделены типы матриц, соответствующие 
паранепротиворечивым, параполным (см. |Томова, 2020]) и паранормальным логикам, а также определены теории, индуцируемые этими матрицами. В настоящее время остается открытым вопрос о функциональных свойствах исследуемого класса $n$-значных матриц.

\section{Литература}

Девяткин, 2016 - Девяткин Л.Ю. Неклассические модификации многозначных матриц классической логики. Ч. I // Логические исследования. 2016. Т. 22. № 2. C. $27-58$.

Томова, 2018 - Томова H.E. О свойствах одного класса четырехзначных паранормальных логик // Логические исследования / Logical Investigations. 2018. T. 24. № 1. C. $75-89$.

Томова, 2020 - Томова H.E. Об одном классе $n$-значных литеральных паранепротиворечивых / параполных логик // Логические исследования / Logical Investigations. 2020. T. 26. № 2. C. 144-159.

Bolc, 1992 - Bolc L., Borowik P. Many-Valued Logics 1: Theoretical Foundations. Springer-Verlag Berlin Heidelberg, 1992. 288 p.

Ciuciura, 2015 - Ciuciura J. A weakly-intuitionistic logic I1 // Logical Investigations. 2015. Vol. 21. No. 2. P. 53-60.

Fernández, 2001 - Fernández V.L. Semântica de Sociedades para Lógicas $n$-valentes. Campinas: IFCH-UNICAMP, 2001. 126 p.

Fernández, Coniglio, 2003 - Fernández V.L., Coniglio M.E. Combining valuations with society semantics // Journal of Applied Non-Classical Logics. 2003. Vol. 13. No. 1. P. 21-46.

Jaskowski, 1969 - Jaśkowski S. A propositional calculus for inconsistent deductive systems // Studia Logica. 1969. Vol. 24. P. 143-157.

Karpenko, 1999 - Karpenko A.S. Jaśkowski's criterion and three-valued paraconsistent logics // Logic and Logical Philosophy. 1999. Vol. 7. P. 81-86.

Lewin, Mikenberg, 2006 - Lewin R.A., Mikenberg I.F. Literal-paraconsistent and literal-paracomplete matrices // Math. Log. Quart. 2006. Vol. 52. No. 5. P. 478-493.

Popov, 1999 - Popov V.M. On the logics related to A. Arruda's system V1 // Logic and Logical Philosophy. 1999. Vol. 7. P. 87-90.

Puga, Da Costa, 1988 - Puga L.Z., Da Costa N.C.A. On the imaginary logic of N.A. Vasiliev // Z. Math. Logik Grundl. Math. 1988. Vol. 34. P. 205-211.

Sette, 1973 - Sette A.M. On propositional calculus P1 // Mathemetica Japonicae. 1973. Vol. 18. P. 173-180.

Sette, Carnielli, 1995 - Sette A.M., Carnielli W.A. Maximal weakly-intuitionistic logics // Studia Logica. 1995. Vol. 55. No. 1. P. 181-203.

Wójcicki, 1984 - Wójcicki $R$. Lectures on Propositional Calculi. Wroclaw: Ossolineum, 1984. 292 p. 


\author{
Natalya E. TOMOVA
}

\title{
On a paranormal criterion for $n$-valued logical matrices
}

\author{
Natalya E. Tomova \\ Institute of Philosophy, Russian Academy of Sciences, \\ 12/1 Goncharnaya Str., Moscow, 109240, Russian Federation. \\ E-mail: natalya-tomova@yandex.ru
}

\begin{abstract}
As a result of generalization of the $n$-valued case of the algorithm for constructing literal paraconsistent / paracomplete logics by combining isomorphs of classical logic, we obtain classes of paraconsistent, paracomplete and paranormal logics. A logic is called paranormal if is it both paraconsistent and paracomplete at the same time.

The non-verifiability of the Duns Scotus law in the corresponding logical matrix is taken as the criterion of the paraconsistency of logic. The non-verifiability of Clavius' law in the corresponding logical matrix is taken as a criterion of the paracompleteness of logic. The paper deals the type of $n$-valued logical matrices that define paranormal systems. The class of tautologies defined by this type of matrices is investigated. It is proved that with respect to the induced class of tautologies, the studied matrices coincide with the four-valued paranormal matrices of the logics $\mathbf{V}, \mathbf{I}^{\mathbf{1}} \mathbf{P}^{\mathbf{1}}$ known in the literature.
\end{abstract}

Keywords: paraconsistency, paracompletness, many-valued logics, logical matrices, isomorphs

For citation: Tomova N.E. "O kriterii paranormal'nosti dlya $n$-znachnykh logicheskikh matrits" [On a paranormal criterion for $n$-valued logical matrices], Logicheskie Issledovaniya / Logical Investigations, 2021, Vol. 27, No. 2, pp. 121-132. DOI: 10.21146/2074-1472-2021-272-121-132 (In Russian)

\section{References}

Devyatkin, 2016 - Devyatkin, L.Yu. "Neklassicheskie modifikatsii mnogoznachnykh matrits klassicheskoi logiki. Chast' I" [Non-classical Modifications of Many-valued Matrices of the Classical Propositional Logic. Part I], Logical Investigations, 2016, Vol. 22, No. 2, pp. 27-58.

Bolc, 1992 - Bolc, L., Borowik, P. Many-Valued Logics 1: Theoretical Foundations. Berlin: Springer-Verlag Berlin Heidelberg, 1992. 288 pp.

Ciuciura, 2015 - Ciuciura, J. "A weakly-intuitionistic logic I1", Logical Investigations, 2015, Vol. 21, No. 2, pp. 53-60.

Fernández, 2001 - Fernández, V.L. Semântica de Sociedades para Lógicas n-valentes. Campinas: IFCH-UNICAMP, 2001. 126 pp. 
Fernández, Coniglio, 2003 - Fernández, V.L., Coniglio, M.E. "Combining valuations with society semantics", Journal of Applied Non-Classical Logics, 2003, Vol. 13, No. 1, pp. 21-46.

Jaskowski, 1969 - Jaśkowski, S. "A propositional calculus for inconsistent deductive systems", Studia Logica, 1969, Vol. 24, pp. 143-157.

Karpenko, 1999 - Karpenko, A.S. "Jaśkowski's criterion and three-valued paraconsistent logics", Logic and Logical Philosophy, 1999, Vol. 7, pp. 81-86.

Lewin, Mikenberg, 2006 - Lewin, R.A., Mikenberg, I.F. "Literal-paraconsistent and literal-paracomplete matrices", Math. Log. Quart., 2006, Vol. 52, No. 5, pp. 478-493.

Popov, 1999 - Popov, V.M. "On the logics related to A. Arruda's system V1", Logic and Logical Philosophy, 1999, Vol. 7, pp. 87-90.

Puga, Da Costa, 1988 - Puga, L.Z., Da Costa, N.C.A. "On the imaginary logic of N.A. Vasiliev", Z. Math. Logik Grundl. Math., 1988, Vol. 34, pp. 205-211.

Sette, 1973 - Sette, A.M. "On propositional calculus P1", Mathemetica Japonicae, 1973, Vol. 18, pp. 173-180.

Sette, Carnielli, 1995 - Sette, A.M., Carnielli, W.A. "Maximal weakly-intuitionistic logics", Studia Logica, 1995, Vol. 55, No. 1, pp. 181-203.

Tomova, 2018 - Tomova, N.E. "O svoistvakh odnogo klassa chetyrekhznachnykh paranormal'nykh logik" [On properties of a class of four-valued paranormallogics], Logical Investigations, 2018, Vol. 24, No. 1, pp. 75-89.

Tomova, 2020 - Tomova, N.E. "Ob odnom klasse n-znachnykh literal'nykh paraneprotivorechivykh/parapolnykh logik" [On a class of $n$-valued literal paraconsistent/paracomplete logics], Logical Investigations, 2020, Vol. 26, No. 2, pp. $144-159$.

Wójcicki, 1984 - Wójcicki, R. Lectures on Propositional Calculi. Wroclaw: Ossolineum, 1984. 292 pp. 